\title{
Efficiency Evaluation Method for EV Drive System in Real Conditions
}

\author{
Zhang Yanyi ${ }^{\mathrm{a}}$,Huang $\mathrm{Xin}^{\mathrm{b}}$,Cai Zhitao ${ }^{\mathrm{c}}$ andWang Renguang ${ }^{\mathrm{d}}$
}

China Automotive Technology and Research Center, No.68Xianfengdong Rd, Dongli Disrict, Tianjin 300300,China

azhangyanyi@catrc.ac.cn, ${ }^{\mathrm{b}}$ huangxin@catrc.ac.cn, ${ }^{\mathrm{c}}$ caizhitao@catrc.ac.cn, ${ }^{\mathrm{d}}$ tianyu97@163.com

Keywords:electric vehicle, motor drive system, efficiency evaluation, BEV development

Abstract. The test requirements and evaluation criteria for efficiency of EV motor drive system were briefly introduced basing on China national standards. Then the difference of efficiency performance was analyzed using data collected from real road condition and test bench. From data of test bench,a pre-evaluation method for pure electric vehicle is formed to estimate the efficiency performance of the electric motor drive system under different testing conditions, which can benefit electric motor matching during EV development period.

\section{Introduction}

With development of electric vehicles, the test and evaluation requirements defined by national standards and regulation for BEVs and main components are becoming more complete, but most of them are focused on performance tests. The matching of e driving system and whole vehicle can influence the performance development of them. Therefore, the test and evaluation of electric motor driving and its main components should be extended to make good use of their test data from test bench, to evaluate their performance development in real vehicle conditions, which can serve the BEV development basing on test data and further analysis.

The efficiency requirements for electric drive motors have been described in related China national standards, the difference of efficiency between the data from BEV and test data of e electric drive motor are obvious after they are integrated in BEVs. In order to evaluation the performance of electric motors after integration with vehicle, a efficiency performance evaluation method is formed basing on their test data, which can be reference tools for BEVs development.

\section{Test Methods forMotor DriveSystem by China Standards}

There are two national standards for electric motor driving system including motor and controller are in use now, which are GB/T18488.1-2015<Electric drive motor system for electric vehicles-Part 1: Specification $>[1]$ and GB/T18488.2-2015<Electric drive motor system for electric vehicles-Part 2: test methods $>$ [2], these two version of 2015 are for replacing the old version of these standards of 2006, which are GB/T18488.1-2006[3] and GB/T18488.2-2006[4] . The version of 2015 have been modified to give more detail descriptions for testing electric motor driving system.

In GB/T18488.1-2006, the efficiencyrequirements for e motor drive system are that:the characteristics of the electric motor and its controller should meet their product descriptions. And only test and calculation methods for torque-speed characteristics are presented in GB/T18488.2-2006 without further description for driving efficiency and detail evaluation methods. But in GB/T18488.1-2015, two detail descriptions for driving efficiency are listed: 1) At nominal voltage, the maximum efficiency of driving system should not less those of value decided by motor make and consumers. 2) At nominal voltage, the percentage of the high efficiency (no less than $80 \%$ )to the whole operation area should not less than those value decided by motor make and 
consumers.

The torque-speed characteristics test methods are also defined in GB/T18488.2-2015, which requires that no less than 10 speed points with at least 10 torque test points for each speed point should be measured (for high speed conditions, the torque points can be reduced according to real conditions). Then at least 100 test points and their correspond efficiency values should be calculated and evaluated according to GB/T18488.1-2015.

Basing on test conducted according to two standards of 2015, the efficiency MAP of electric drive motor can be gotten easily, which can describe the efficiency characteristics of electric drive motor with presentation of maximum efficiency and high efficiency working area. But in practical conditions, the electric motor performance can be affected by road condition, parameter matching and control strategy, which make the maximum efficiency and high efficiency work area different from test data.

\section{Analysis on Test Efficiency and Real Operation Efficiency of Electric Drive Motor}

In order to further understanding the efficiency differences under different conditions, One BEV vehicle was adopted to conducted tests according China national standards firstly, then the real tests were performed using NEDC cycle and several different real roads in Tianjin. The following parts are based on these data collected from several conditions.

The figure one is the results of efficiency of electric drive motor measured according to GB/T18488.1-2015 and GB/T18488.2-2015. In driving mode, its top speed is 6200rpm, maximum torque is $108 \mathrm{~N} \cdot \mathrm{m}$, top efficiency is $93.89 \%$ and its high efficiency area percentage is $91.21 \%$; In generating mode, its top speed is $6200 \mathrm{rpm}$, maximum torque is $105 \mathrm{~N} \cdot \mathrm{m}$, top efficiency is $94.59 \%$ and its high efficiency area percentage is up to $87.9 \%$.

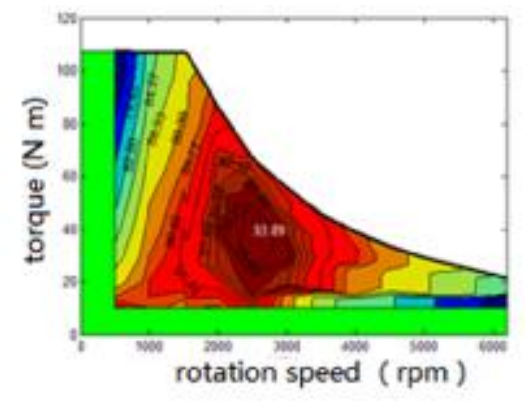

(a) drive mode

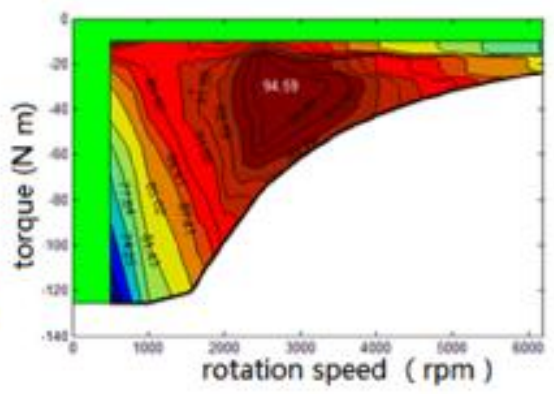

(b) generation mode

Figure 1Efficiency MAP of drive system

The speed - time profiles of NEDC cycle and real road conditions are shown in figure 2. 


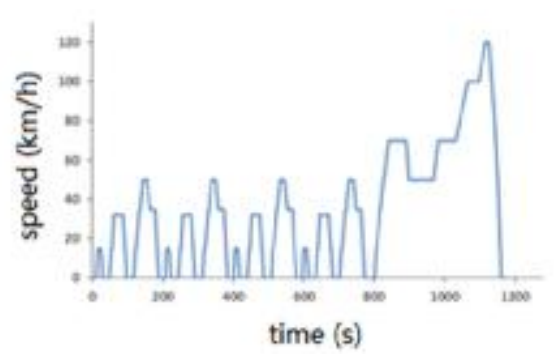

(a) Speed profile NEDC cycle

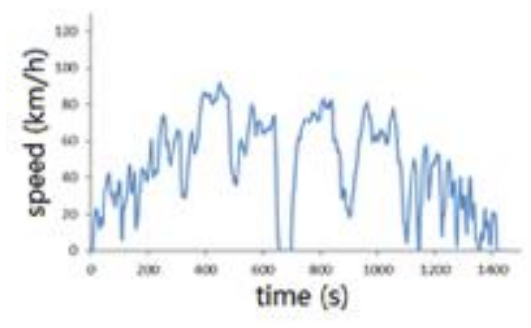

(c)Speed profile -suburban Tianjin

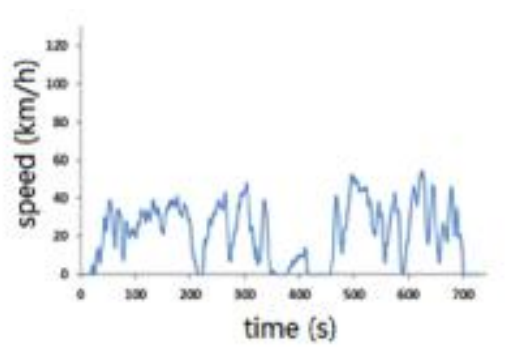

(b)Speed profile -urban in Tianjin

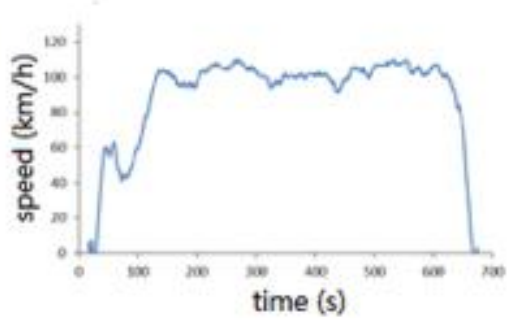

(d)Speed profile-freeway Tianjin

Figure 2Speed profile under real road conditions

Under these four different cycle and road conditions, the operation points of motor are shown in figure 3, which were derived from onboard data acquisition system. From these figures, we can find that the electric motor speeds are less than 4000rom, torque values ranges $\pm 80 \mathrm{~N} \cdot \mathrm{m}$, which are all smaller than those value shown in figure one.

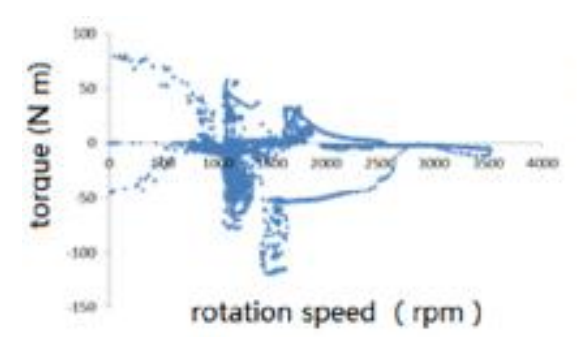

(a)data distribution in NEDC

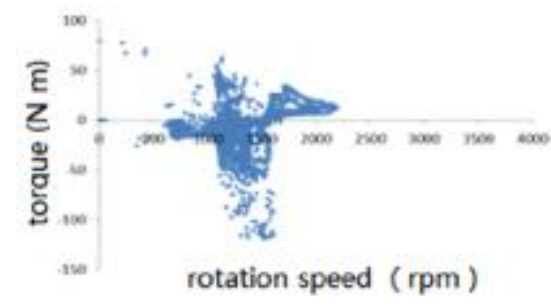

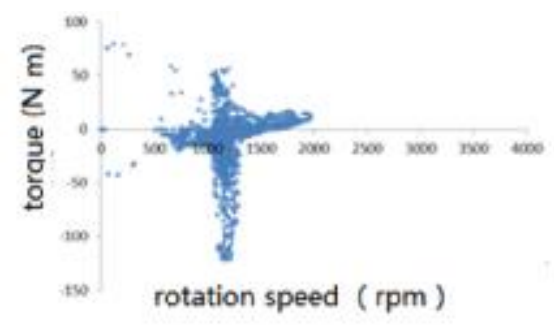

(b)data distribution in urban Tianjin

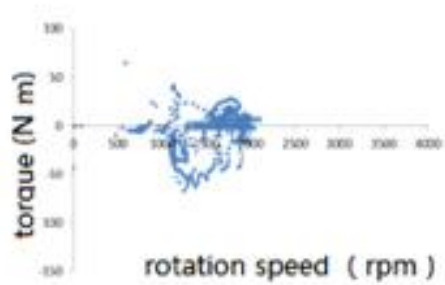

(c)data distribution in suburban Tianjin (d)data distribution in freeway Tianjin

Figure 3 working points distribution under different real conditions

The interpolation method was adopted to get efficiency values under working points of above mentioned four basing on efficiency MAP in figure one. The results are shown in figure four. 

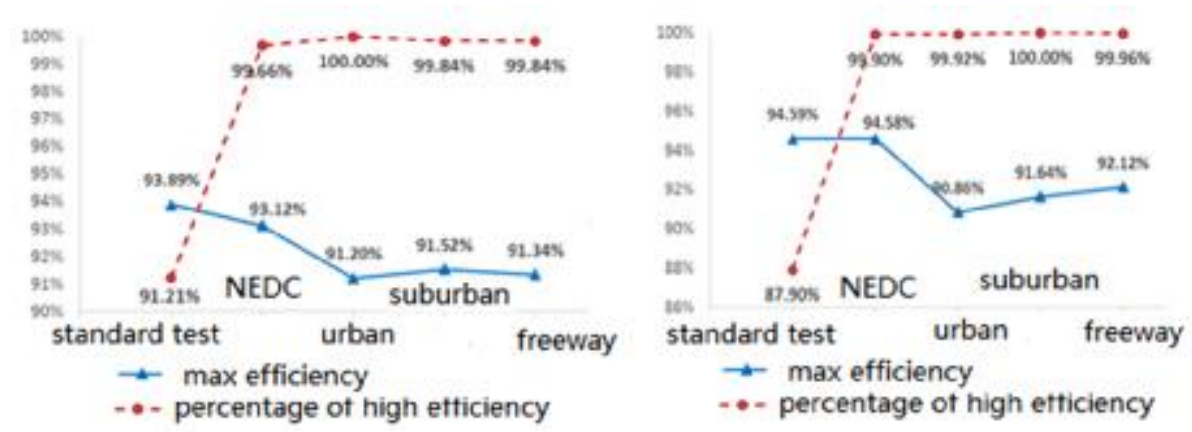

(a)efficiency under drive mode (b) efficiency under generation mode

Figure 4 efficiency of electric drive motor in four real conditions

As shown in figure 4, comparison the results after integration of BEV with the values gotten from bench tests, the maximum efficiency is smaller, but these working points are almost all located in high efficacy area. These results show that, although the electric motor cannot reach its top efficiency, its control strategy can make it operated in high efficiency area to save energy.

From this analysis, we can understand that the electric motor performance conditions in real BEVs basing on the efficiency characteristics of motor system. But just from the tests results under bench test required by china standards, it is difficult to know the operation data under real road conditions.

If there is a reliable method which can be used to pre evalutate the perfromance of motor system, it will benefit BEV development process. Before the electric motor matching results are confirmed,basing on these data from satandard tests, the engineesr can compare the perfromance for sevearl candidate electric motors to decide which one is suitable for BEVs best.

\section{EvaluationMethods for Electric Drive MotorSystem in BEVS}

The electric motors are only power source for BEVs. The distribution of operating points under different conditions can be derived from simulation calculation basing on the vehicles dynamics principals and its basic specification, and then we can get the efficiency performance with higher accuracy for matching of motor system with whole vehicle. In figure 5, the efficiency MAP of one electric motor system used in a BEV is presented under drive mode with maximum efficiency $96.13 \%$ and high efficiency area $88.89 \%$.

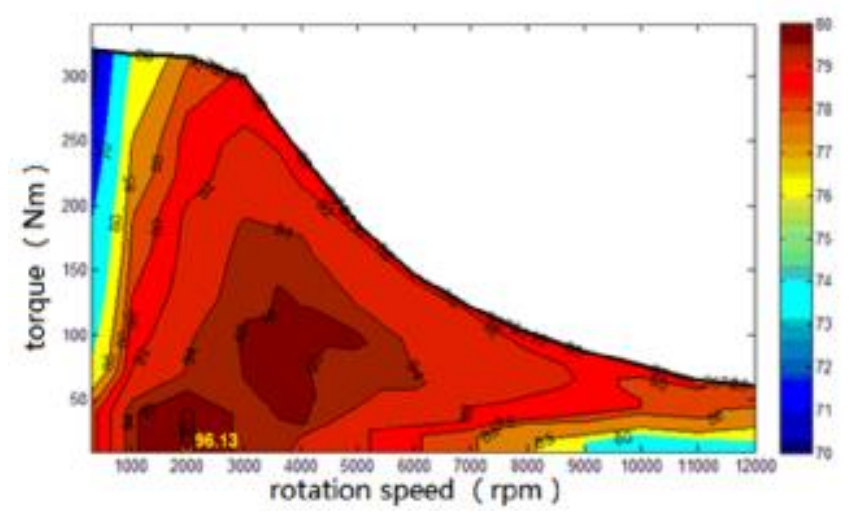

Figure 5 Efficiency Map of drive system under drive mode

From the vehicle force balance analysis ${ }^{[5]}$, equation 1 is shown as following:

$$
F_{\text {drv }}-F_{\text {brk }}-\left(m g f \cos \left(\arctan \frac{i}{100}\right)+m g \sin \left(\arctan \frac{i}{100}\right)+\frac{C_{\mathrm{D}} A v^{2}}{21.15}\right)=\delta m \frac{\mathrm{d} v}{\mathrm{~d} t}
$$




$$
\begin{gathered}
T_{m o t}=F_{d r v} r / i_{0}(2) \\
n_{m o t}=v i_{0} /(0.377 r)(3)
\end{gathered}
$$

Where, $F_{\mathrm{drv}}$ is driving force, $F_{\mathrm{brk}}$ is braking force, $m$ is vehicle mas, $r$ is rolling radius of wheel, $\mathrm{g}$ is gravity acceleration, fis rolling resistance coefficient, $i$ is grade, $C_{\mathrm{D}}$ is drag coefficient, $A$ is frontal area, $\nu$ is vehicle speed, $\delta$ is auto rotating mass conversion factor, $i_{0}$ is transmission ratio, $T_{\text {mot }}$ is motor torque, $n_{\text {mot }}$ is motor speed.

According to practical parameters of the $\mathrm{BEV}$ matched with this motor, in motor drive mode, let $F_{\text {brk }}=0, i=0, m=3000 \mathrm{~kg}, f=0.0120, A=5.07 \mathrm{~m}^{2}, C_{\mathrm{D}}=0.55, \delta=1.04, i_{0}=9.98, r=0.364 \mathrm{~m}$. And then the working points of motor, under 4 different conditions shown in figure 2 , are calculated and the results are presented in figure 6.
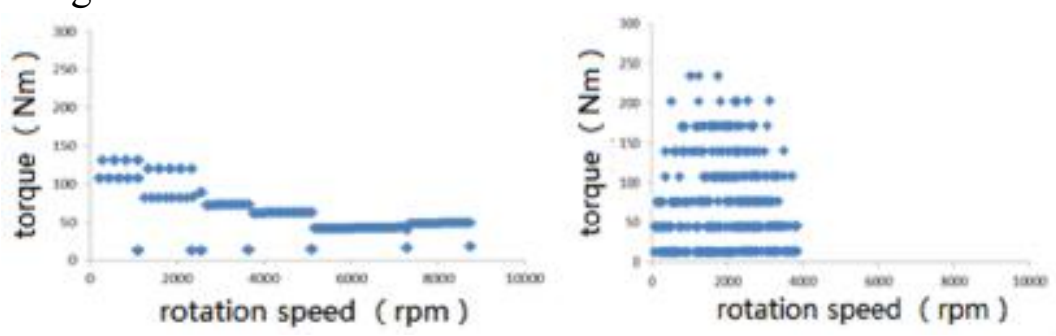

(a) data distribution in NEDC cycle

(b) data distribution in urban Tianjin

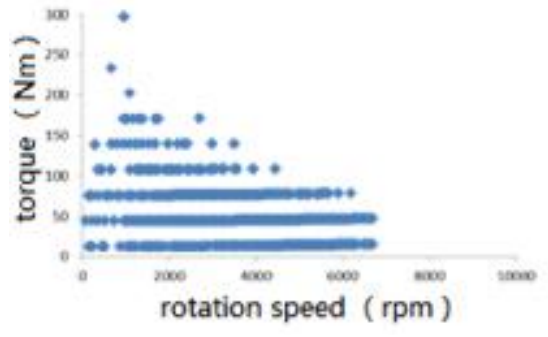

(c) data distribution in suburban Tianjin

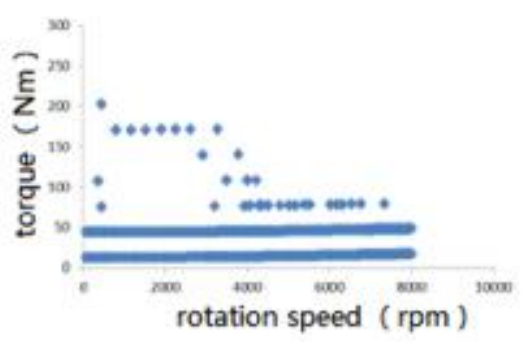

(d) data distribution in freeway Tianjin

Figure 6 motor work point distribution under 4 conditions

After the working points were gotten from 4 different conditions, then calculated the corresponding efficiency values using interpolation method basing on the efficiency MAP presented in figure 5, which are the pre-evaluated efficiency of motor driving system and shown in figure 7. From figure 7, we can find that, after electric drive motor matched properly with the BEV, it can reach its maximum efficiency and the high efficiency area can be improved and maintained above $96.40 \%$. It shows that the electric drive motor produces a satisfied performance of efficiency after good matching using thispre-evaluation method.

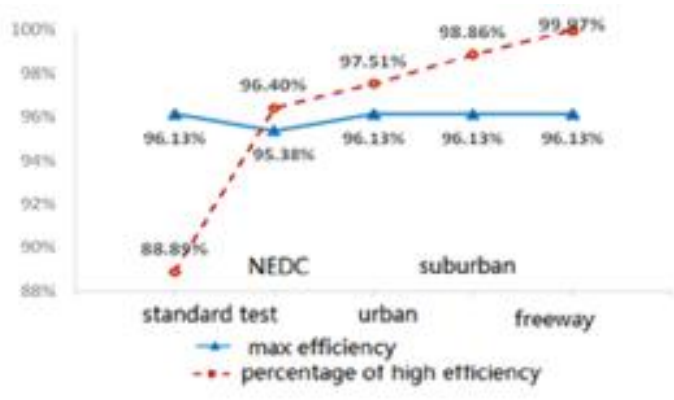

Figure 7evaluation results under 4 driving conditions

According to this pre-evaluation method, the electric motor efficiency after matching with whole vehicle can be derived using some basic parameters in early time of development process, which 
can benefit the electric motor choice, matching process and control strategy development.

\section{Conclusions}

On the basis of analysis of China standards on electric motor driving system for electric vehicles, a $\mathrm{BEV}$ vehicle was tested in real road conditions to get practical operation data, and then compare the efficiency difference between the data from the bench test and real road test. Then a pre-evaluation method was put forward to calculate efficiency of motor drive in real road conditions. This method can be used in early development process with few parameters and motor test data, which make good use of motor test data to support motor matching and BEV development and benefit the BEV test and development.

\section{References}

[1] GB/T18488.1-2015.Electric drive motor system for electric vehicles-Part 1: Specification [S].Beijing: China Standard Press,2015

[2] GB/T18488.2-2015.Electric drive motor system for electric vehicles-Part 2: Test method [S].Beijing: China Standard Press,2015

[3] GB/T18488.1-2006.Electric drive motor system for electric vehicles-Part 1: Specification [S]. Beijing: China Standard Press,2006

[4] GB/T18488.2-2006.Electric drive motor system for electric vehicles-Part 2: Test method [S]. Beijing: China Standard Press,2006

[5] Liu Zhaodu .Automotive Science [M].Beijing: High Education Press,2012.9 